\title{
Vaccination against pneumococcus in West Africa: perspectives and prospects
}

This article was published in the following Dove Press journal:

International Journal of General Medicine

10 September 2013

Number of times this article has been viewed

Eric S Donkor'

Nicholas TKD Dayie ${ }^{1,2}$

Ebenezer $V$ Badoe $^{3}$

'Department of Microbiology, University of Ghana Medical

School, Accra, Ghana; ${ }^{2}$ Department of Veterinary Disease Biology,

Faculty of Health and Medical Sciences, University of Copenhagen, Copenhagen, Denmark; ${ }^{3}$ Department of Child Health, University of Ghana Medical School, Accra, Ghana
Correspondence: Eric S Donkor Department of Microbiology, University of Ghana Medical School, PO Box 4236, Accra, Ghana Email eric.sampane-donkor@Ishtm.ac.uk
Background: Pneumococcal vaccination has become obligatory due to the enormous burden of pneumococcal diseases. Quite recently, pneumococcal conjugate vaccines have been developed, and have been shown to be superior to the previous polyvalent polysaccharide vaccine of the organism. Pneumococcal conjugate vaccines (PCVs) are being introduced in many West African countries and it is important to understand the expected performance, relevance, and limitations of these vaccines in the subregion.

Aim: The objective of the study presented here was to provide epidemiological insights into PCVs in West Africa based on the prevailing pneumococcal serotypes in the subregion.

Methods: A systematic review was carried out on pneumococcal serotypes causing invasive and noninvasive diseases in West Africa. Studies included in the review were those that reported at least 20 serotyped pneumococcal isolates and which were conducted prior to the introduction of PCVs in the region in 2009. The proportion of pneumococcal disease associated with each serotype as well as the serotype coverage of various PCVs (PCV7, PCV10, and PCV13) were calculated.

Results: The data covered 718 serotyped pneumococcal isolates from six West African countries: Burkina Faso, Ghana, Nigeria, Mali, Senegal, and The Gambia. The 718 isolates covered more than 20 serotypes. Serotype 1 was the most prevalent serotype (32\%), followed by serotype $5(15 \%)$, serotype $6(7 \%)$, serotype $2(6 \%)$, serotype $3(6 \%)$, and serotype $12(5 \%)$. The estimated serotype coverage of PCVs among the West African countries was $2 \%-36 \%$ for PCV7, 39\%-80\% for PCV10, and 65\%-87\% for PCV13.

Conclusion: A pneumococcal capsular vaccine for use in West Africa must contain serotypes 1 and 5, the most important serotypes responsible for pneumococcal disease in the region. Consequently, while PCV10 and PCV13 are generally suitable for use in West Africa, PCV7 is unsuitable.

Keywords: pneumococcus, conjugate vaccines, serotype, PVC10, PVC13, pneumococcal disease, Streptococcus pneumoniae

\section{Introduction}

Pneumococcus is a major cause of several invasive and noninvasive diseases including pneumonia, meningitis, septicemia, sinusitis, and acute otitis media. Though a global problem, pneumococcal disease is more of a burden in the developing world, where children less than 5 years old are most affected, and the organism is responsible for $10 \%-20 \%$ of all deaths in this age group. ${ }^{1,2}$ The burden of pneumococcal diseases is further compounded by the trend of increasing antibiotic resistance exhibited by the organism, especially multiple drug resistance. ${ }^{3}$ 
Thus, pneumococcal vaccines have become obligatory owing to the enormous problem posed by pneumococcal disease. At present, two types of pneumococcal vaccines are available, the polysaccharide (unconjugated) vaccine and the recently developed conjugate vaccines (Table 1). The polysaccharide vaccine has 23 serotypes, and has an average good efficacy in protecting against these serotypes of $\sim 60 \%-70 \%{ }^{1}$ However, its usefulness is limited in the elderly and immunocompromised individuals, as the duration of protection is short. ${ }^{1}$ Additionally, infants respond poorly to the vaccine and it induces no herd effect. ${ }^{1}$ Most of these limitations have been overcome by the newly introduced conjugate vaccines, in which purified capsular polysaccharides are conjugated to a nontoxic variant of diphtheria toxin. Pneumococcal conjugate vaccines (PCVs) consist of 7-13 capsular serotypes of Streptococcus pneumoniae and have been shown to have a high efficacy against infections in children. ${ }^{1,4-6}$ In addition, the conjugate vaccines have a relatively higher potential to control the serotypes most commonly associated with multiple drug resistance. ${ }^{1}$

In West Africa, pneumococcal diseases and outbreaks are relatively common, ${ }^{7-10}$ which may partly be due to the high pneumococcal carriage rates in West Africa and the prevalence of risk factors such as overcrowding that favor transmission of the organism. ${ }^{10-14}$ Given the impact of pneumococcal diseases in West Africa, the importance of introducing routine pneumococcal vaccination in the region cannot be overemphasized. Massive vaccination with PCVs,

Table I Features of Streptococcus pneumoniae polysaccharide and conjugate vaccines

\begin{tabular}{lll}
\hline Vaccine feature & $\begin{array}{l}\text { Polysaccharide } \\
\text { vaccines } \\
\text { (unconjugated) }\end{array}$ & $\begin{array}{l}\text { Conjugate } \\
\text { vaccines (protein } \\
\text { + saccharide) }\end{array}$ \\
\hline $\begin{array}{l}\text { Efficacy for prevention of } \\
\text { invasive disease in infants } \\
<2 \text { years old' }\end{array}$ & No & Yes \\
$\begin{array}{l}\text { Vaccine licensed for usage } \\
\text { in infants }<2 \text { years old }\end{array}$ & No \\
$\begin{array}{l}\text { Ability to prevent invasive } \\
\text { disease in adults and } \\
\text { children }>2 \text { years old }\end{array}$ & Yes & Yes \\
$\begin{array}{l}\text { Recommended for usage } \\
\text { in high-risk populations }{ }^{1,6}\end{array}$ & Yes & Yes \\
$\begin{array}{l}\text { Ability to generate T-cell } \\
\text { memory }\end{array}$ & No \\
$\begin{array}{l}\text { Ability to prevent } \\
\text { colonization with vaccine } \\
\text { serotypes' }\end{array}$ & No & Yes \\
$\begin{array}{l}\text { Number of serotypes } \\
\text { in vaccine, }\end{array}$ & 23 & Yes \\
\hline
\end{tabular}

particularly among children, is expected in West Africa, as many countries in the subregion have officially expressed interest in introducing the vaccines into their national childhood immunization programs. ${ }^{15}$ In view of this, it is important to understand the expected performance, relevance, and limitations of the vaccines in a West African context, based on the prevailing pneumococcal serotypes in the West African region. In this paper, we provide epidemiological insights into PCVs in West Africa, thus providing policyrelevant information and directions for future research on the subject.

\section{Methods}

The study reported here employed a systematic review approach using the Preferred Reporting Items for Systematic Reviews and Meta-Analyses (PRISMA) guidelines. ${ }^{16}$ A search was conducted in relevant databases to identify, locate, and retrieve the required data for the study. The keywords "Streptococcus pneumoniae" or "pneumococcus" were used to review all references on $S$. pneumoniae that were geographically linked to West African countries, and databases searched included PubMed, ISI Web of Knowledge, and African Journals Online. In the study reported here, the definition of "West Africa" was based on the United Nations' subregional grouping. The inclusion criteria of the systematic review were articles that reported pneumococcal serotype data from invasive or noninvasive disease cases in West Africa and which were conducted prior to the introduction of PCVs in the region in 2009. The systematic review was not limited to any age group or specific demographic features, but articles that investigated selected serotypes or reported fewer than 20 serotyped pneumococcal isolates were excluded.

Data extracted from the articles that fulfilled the inclusion criteria of the study were pneumococcal serotype, patient age, patient sex, clinical diagnosis, and country. Analysis of the data involved descriptive statistics, while significant associations of relevant variables were assessed at a level of $P<0.05$. The proportion of pneumococcal disease associated with each serotype as well as the serotype coverage of various PCVs (PCV7, PCV10, and PCV13) were calculated. The contribution of each pneumococcal serotype to disease was calculated independent of the other serotypes. The relationship between pneumococcal serotypes and different age groups was investigated, but this was limited to each country's data due to the extensive heterogeneity of age groups reported by the various articles used in the study. In calculating the vaccine coverage of pneumococcal 
serotypes, data that reported serogroups rather than specific serotypes were excluded. The serotype coverage of a PCV formulation was defined as the sum of the proportion of serotypes of the vaccine type. Vaccine serotype coverage was multiplied by the incidence and mortality estimates for pneumococcal disease to estimate vaccine impacts for the various PCV formulations.

\section{Results}

Figure 1 is a flowchart summarizing article retrieval, as well as stepwise inclusion and exclusion of articles in the systematic review. A total of 15 original research articles have reported on serotypes of pneumococcal diseases in West Africa: three from Ghana, three from Burkina Faso, two from Nigeria, two from Senegal, one from Mali, and four from The Gambia. Nine of the articles met the inclusion criteria of the systematic review, but three of these were excluded as they investigated or reported selected serotypes (Table 2). The six studies included in the systematic review cover seven West African countries (Burkina Faso, Togo, Senegal, Ghana, The Gambia, Nigeria, and Mali), span a period of 27 years (1978-2005), and involved 1,970 pneumococcal disease cases and 932 pneumococcal mortality cases (inferred from Table 2).

The systematic review involved $718 \mathrm{~S}$. pneumoniae serotyped isolates representing the six countries of data collection (Tables 2 and 3). As shown in Table 3, over

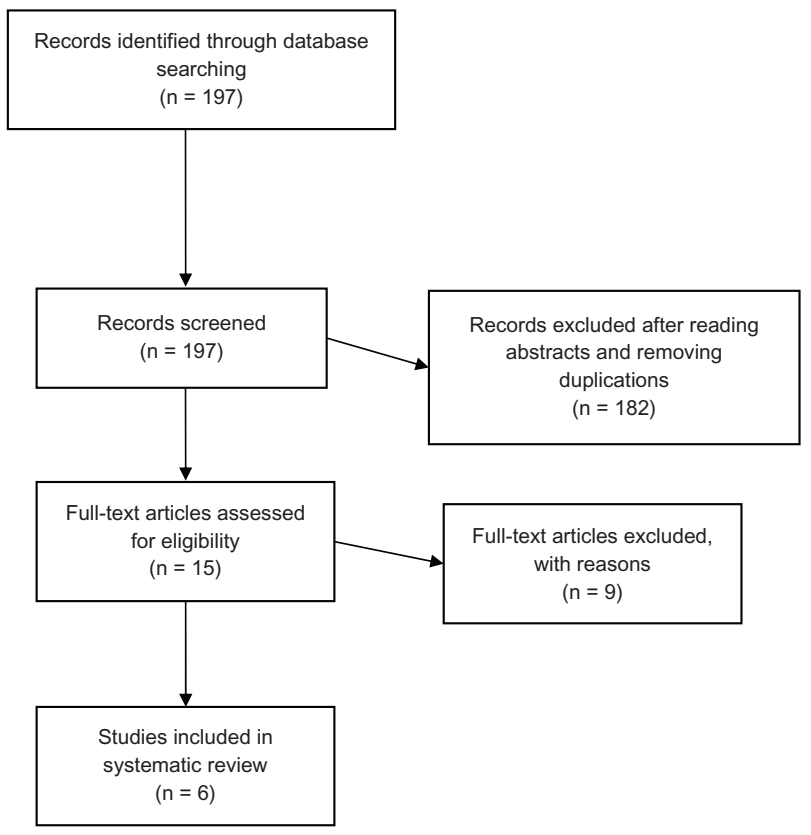

Figure I Preferred Reporting Items for Systematic Reviews and Meta-Analyses (PRISMA) flow diagram of the systematic review.
30 pneumococcal serotypes are involved in disease in West Africa and between eight and 28 serotypes may be represented in a country. Serotype 1 was the most prevalent serotype in pneumococcal disease, accounting for $32 \%$ of the serotypes that caused various diseases, followed by serotype 5 (15\%). Serotypes 1 and 5, along with serotypes/serogroups $6(7 \%)$, $2(6 \%), 3(6 \%)$, and $12(5 \%)$, represented a prevalence of $71 \%$. In children (usually $<5$ years), the most important serotypes of invasive disease were $5,6 \mathrm{~A}, 1$, and 14 , while, in older people, serotype 1 was the main serotype associated with invasive disease. Serotypes 23, 19, and 12 tend to have limited geographical distribution in West Africa, but could be quite prevalent in the countries where they occurred. A large number of serotypes/serogroups (20, 21, 22A, 24A, 29, 33F, $35,38,40,45,46$, and 48$)$ had very low prevalence $(<1 \%)$ in disease and very limited geographical distribution, occurring usually in one country. Comparison of the top three serotypes country by country revealed that the top three serotypes in Burkina Faso were the same as the top three serotypes in Senegal: serotypes 1,5 , and 6 . The top three serotypes in Ghana $(1,3$, and 8$)$ were similar to those in Nigeria (1,3, and 5). The top three serotypes in The Gambia (14, 12F, and 19A) and in Mali $(5,2$, and $7 F)$ were more diverse from the serotypes in other West African countries.

The serotype coverage of three PCVs (PCV7, PCV10, and PCV13), based on the serotype distribution data of the articles included in the systematic review, is shown in Figure 2. The overall serotype coverage for the vaccines in West Africa was 12\% for PCV7, 68\% for PCV10, and 79\% for PCV13. Across the different West African countries, serotype coverage for PCV7 was 2\%-36\%, serotype coverage for PCV10 was 39\%-80\%, and serotype coverage for PCV13 was $65 \%-87 \%$. The highest serotype coverage of PCV10 and PCV13 occurred in Ghana. The Gambia had the highest serotype coverage of PCV7 and also the least serotype coverage of PCV13. The least serotype coverage of PCV7 and PCV10 occurred in Nigeria and Burkina Faso, respectively. Generally, for each conjugate vaccine type, there were no significant differences in serotype coverage among the different West African countries at $P<0.05$.

As can be inferred from Table 2, there were 1,970 pneumococcal disease cases and 932 pneumococcal mortality cases; the estimated public health impact of PCVs based on these data is illustrated in Figure 3. A total of 236 of the 1,970 cases were caused by PCV7 serotypes; the additional serotypes in PCV10 and PCV13 were responsible for 1,104 (1,340 minus 236) cases and 1,320 (1,556 minus 236) cases, respectively. A total of 112 pneumococcal mortality cases 
Table 2 Epidemiological features of studies that satisfied inclusion criteria of the systematic review

\begin{tabular}{|c|c|c|c|c|c|c|c|c|}
\hline Study & Country & ST & Period & $\begin{array}{l}\text { Age of } \\
\text { subjects, y }\end{array}$ & Setting & Specimen(s) & $\begin{array}{l}\text { Pneumo } \\
\text { cases }\end{array}$ & $\begin{array}{l}\text { Pneumo } \\
\text { deaths }\end{array}$ \\
\hline Traore et $\mathrm{al}^{27, *}$ & B Faso/Togo & PT & $2002-2006$ & $\mathrm{C} / \mathrm{A}$ & Rural and urban & CSF & 463 & 171 \\
\hline Cadoz et $\mathrm{al}^{28}$ & Senegal & RT & $1970-1979$ & $<2$ & Urban & CSF & 983 & 583 \\
\hline Leimkugel et $\mathrm{a}^{29}$ & Ghana & PT & 1998-2003 & $\mathrm{C} / \mathrm{A}$ & Rural & CSF & 117 & 51 \\
\hline Enwere et $\mathrm{al}^{30}$ & Gambia & PT & $2000-2004$ & $<2.5$ & Rural & CSF, blood, aspirates & 116 & 19 \\
\hline Onyemelukwe and & Nigeria & PT & $1978-1980$ & $<5$ & Urban & CSF, aspirates, sputum & 300 & 83 \\
\hline \multicolumn{9}{|l|}{ Greenwood ${ }^{31}$} \\
\hline Campbell et a ${ }^{32}$ & Mali & PT & $2002-2003$ & $<5$ & Urban & CSF, blood, aspirates & 106 & 25 \\
\hline Adegbola et $\mathrm{al}^{33, * *}$ & Gambia & PT & $1996-2003$ & $\mathrm{C} / \mathrm{A}$ & Rural and urban & CSF, blood, aspirates & 531 & 55 \\
\hline Antonio et $\mathrm{a}^{34, * *}$ & Gambia & RT & $1996-2005$ & $\mathrm{C} / \mathrm{A}$ & Rural and urban & CSF, blood, aspirates & 127 & None stated \\
\hline Yaro et $\mathrm{al}^{35, * *}$ & B Faso & PT & $2002-2005$ & $\mathrm{C} / \mathrm{A}$ & Rural & CSF & 249 & 115 \\
\hline
\end{tabular}

Notes: *Serotyping was limited to isolates from Burkina Faso; no isolates from Togo were serotyped; **excluded from systematic review due to the study investigating or reporting selected serotypes.

Abbreviations: B Faso, Burkina Faso; C/A, children/adults; CSF, cerebrospinal fluid; pneumo, pneumococcus disease; PT, prospective; RT, retrospective; ST, surveillance type; $y$, years.

were caused by PCV7 serotypes, while the extra serotypes in PCV10 and PCV13 caused 522 (634 minus 112) deaths and 624 (736 minus 112) deaths, respectively.

\section{Discussion}

Based on data analyzed in this study from various West African countries, pneumococcal serotypes causing disease vary from country to country, however two major serotypes $(1,5)$ and four minor serotypes $(2,6,3$, and 12) tend to occur in the subregion. Across all Africa, serotypes 1, 5, 6A, 6B, 14, $19 \mathrm{~A}, 19 \mathrm{~F}$, and $23 \mathrm{~F}$ have been identified as the major causes of pneumococcal disease. ${ }^{17}$ The burden of pneumococcal disease is relatively greater in children less than 5 years old, and, as shown in this West African study, many more pneumococcal serotypes are involved in pediatric disease than in older people, making the control of pneumococcal disease in the former more difficult. The major serotypes causing pediatric pneumococcal disease in West Africa (6A, 5, 14, 1 , and 3) are generally similar to those that do so in other regions in Africa, ${ }^{18,19}$ but may contrast considerably with the pediatric serotypes $(6 \mathrm{~B}, 19 \mathrm{~F}, 18 \mathrm{C}, 23 \mathrm{~F}, 4$, and $9 \mathrm{~V})$ in nonAfrican regions, such as Europe and the USA..$^{20,21}$

Currently, PCVs are being introduced in several West African countries, and it is encouraging that up to $79 \%$ of the pneumococcal serotypes responsible for various diseases in the subregion may be covered by these vaccines. It is worthwhile to discuss the vaccines individually, as they have been made available at different times and because only one vaccine type may be used in a country.

PCV7 was the first of the three vaccines to be introduced in developing countries in 2008., ${ }^{422}$ Prior to this, it had been in use in some industrialized countries, such as the UK, the USA, and several European countries. Globally, PCV7 is estimated to be able to address $54 \%-75 \%$ of invasive pneumococcal disease among children less than 5 years old, with the lowest estimated coverage in Asia and Africa. ${ }^{22}$ In West Africa, the low serotype coverage of PCV7 (2\%-36\%), as observed in this study, is because the vaccine does not contain two of the most prevalent serotypes, 1 and 5, which contribute to severe pneumococcal disease in the subregion. As such, it is clear that this vaccine may not be the most suitable choice for West African countries. PCV7 has actually now been discontinued and is no longer in use. However, the data on PCV7 in this study are important as they illustrate the key point that a pneumococcal capsular vaccine without serotypes 1 and 5 is likely to be of little benefit in the West African region.

PCV10 was licensed in 2008 and introduced in 2010. ${ }^{22}$ As observed in this study, in West Africa, the serotype coverage of PCV10 (39\%-80\%) is considerably higher than that of PCV7 (2\%-36\%), which is mainly due to it including serotypes 1 and 5 .

PCV13 was licensed in 2009 and made available in developing countries in 2012., ${ }^{3,2}$ PCV13 has an appreciably high coverage in West Africa (65\%-87\%) because, unlike PCV10, as well as serotypes 1 and 5, this vaccine also contains serotypes 3 and $6 \mathrm{~A}$, which are prominent in pneumococcal disease in some West African countries.

Cross-protection against serotype 6A is provided by serotype 6B, which is contained in both PCV10 and PCV13. Thus, the practical difference in serotype coverage between PCV10 and PCV13 in West Africa would be more associated with serotype 3 . In this study, serotype 3 occurred in Ghana, Senegal, and Nigeria but not in Burkina Faso, Mali, or The Gambia. Thus, in West African countries where serotype 3 is absent or not prominent in invasive disease, PCV10 may be a 
Table 3 Streptococcus pneumoniae serotypes isolated from various diseases cases in West Africa

\begin{tabular}{|c|c|c|c|c|c|c|c|c|c|c|}
\hline \multirow[b]{2}{*}{ Age, y } & \multicolumn{2}{|c|}{ Burkina Faso } & \multicolumn{2}{|l|}{ Senegal } & \multicolumn{2}{|l|}{ Ghana } & \multirow{2}{*}{$\begin{array}{l}\text { Gambia } \\
<2.5\end{array}$} & \multirow{2}{*}{$\begin{array}{l}\text { Nigeria } \\
<\mathbf{5}\end{array}$} & \multirow{2}{*}{$\frac{\text { Mali }}{<5}$} & \multirow[t]{2}{*}{ Total } \\
\hline & $<5$ & $\geq 5$ & $<2$ & $\geq 2$ & $<5$ & $\geq 5$ & & & & \\
\hline \multicolumn{11}{|c|}{ Serotype } \\
\hline 1 & $3(17 \%)$ & $18(60 \%)$ & $10(9 \%)$ & $90(63 \%)$ & $2(33 \%)$ & $51(80 \%)$ & $8(6 \%)$ & 45 (35\%) & $2(2 \%)$ & 229 (32\%) \\
\hline 2 & $3(17 \%)$ & & $15(14 \%)$ & $3(2 \%)$ & & & $\mathrm{I}(<\mathrm{I} \%)$ & 7 (5\%) & $13(14 \%)$ & $42(6 \%)$ \\
\hline 3 & & & $6(5 \%)$ & $8(6 \%)$ & $2(33 \%)$ & $2(3 \%)$ & & 25 (19\%) & & $40(6 \%)$ \\
\hline 4 & & & & & & & $3(2 \%)$ & $2(2 \%)$ & & $5(<1 \%)$ \\
\hline 5 & $3(17 \%)$ & & $23(21 \%)$ & $8(6 \%)$ & & & II (9\%) & $12(9 \%)$ & $52(54 \%)$ & $109(15 \%)$ \\
\hline 6 & & & 14 (13\%) & $13(9 \%)$ & & & & & $3(3 \%)$ & 48 (7\%)* \\
\hline $6 \mathrm{~A}$ & $5(28 \%)$ & & & & & I (2\%) & $6(5 \%)$ & & & \\
\hline $6 B$ & I $(6 \%)$ & & & & & & $5(4 \%)$ & & & \\
\hline 7 & & I (3\%) & & & & & & & & 12 (2\%) \\
\hline $7 F$ & & & & & & I (2\%) & & & $10(10 \%)$ & \\
\hline 8 & & & & & & $4(6 \%)$ & $2(2 \%)$ & & & $6(<1 \%)$ \\
\hline 9 & & & $6(5 \%)$ & $2(1 \%)$ & & & & I (< I\%) & & $21(3 \%)^{*}$ \\
\hline 9L & & & & & & & $5(4 \%)$ & & & \\
\hline $9 \mathrm{~V}$ & & & & & & & $4(3 \%)$ & & $3(3 \%)$ & \\
\hline 10 & I (6\%) & & & & & & & I (< I\%) & & $3(<1 \%)^{*}$ \\
\hline IOF & & & & & & I (2\%) & & & & \\
\hline II & & & & & & & & $\mathrm{I}(<\mathrm{I} \%)$ & & I (<I\%) \\
\hline 12 & & & & & & & & $3(2 \%)$ & & $35(5 \%)^{*}$ \\
\hline $12 \mathrm{~A}$ & & I (3\%) & $9(8 \%)$ & $6(4 \%)$ & & & & & & \\
\hline I2B & & I (3\%) & & & & & $\mathrm{I}(<\mathrm{I} \%)$ & & & \\
\hline $12 \mathrm{~F}$ & & & & & & $2(3 \%)$ & $12(10 \%)$ & & & \\
\hline 14 & & $2(7 \%)$ & $5(5 \%)$ & $2(1 \%)$ & $2(33 \%)$ & I (2\%) & $20(16 \%)$ & & I (I\%) & $33(5 \%)$ \\
\hline 15 & & & & & & & & $\mathrm{I}(<\mathrm{I} \%)$ & & I (<I\%) \\
\hline $16 \mathrm{~F}$ & & & & & & & $4(3 \%)$ & & & $4(<1 \%)$ \\
\hline 17 & & & & & & & & $3(2 \%)$ & & $3(<2 \%)$ \\
\hline 18 & & & $4(4 \%)$ & $2(1 \%)$ & & & & 7 (5\%) & & $15(2 \%)^{*}$ \\
\hline $18 \mathrm{C}$ & & & & & & & $\mathrm{I}(<\mathrm{I} \%)$ & & & \\
\hline $18 \mathrm{~F}$ & & & & & & & $\mathrm{I}(<\mathrm{I} \%)$ & & & \\
\hline 19 & & & & & & & & $4(3 \%)$ & & $36(5 \%)^{*}$ \\
\hline 19A & & I (3\%) & $4(4 \%)$ & $4(3 \%)$ & & & $12(10 \%)$ & & & \\
\hline $19 \mathrm{~F}$ & & I (3\%) & & & & & $2(2 \%)$ & & $8(8 \%)$ & \\
\hline 20 & & & & & & & $\mathrm{I}(<\mathrm{I} \%)$ & & & $\mathrm{I}(<\mathrm{I} \%)$ \\
\hline 21 & I (6\%) & & & & & & $2(2 \%)$ & $\mathrm{I}(<\mathrm{I} \%)$ & & $4(<1 \%)$ \\
\hline $22 \mathrm{~A}$ & & & & & & & $2(2 \%)$ & & & $2(<1 \%)$ \\
\hline 23 & & & 14 (13\%) & $5(3 \%)$ & & & & $2(2 \%)$ & & $32(5 \%)^{*}$ \\
\hline $23 \mathrm{~F}$ & & & & & & & II (9\%) & & & \\
\hline $24 \mathrm{~A}$ & I (6\%) & & & & & & & & & I (< I\%) \\
\hline 25 & & & & & & & & $3(2 \%)$ & & $7(1 \%)^{*}$ \\
\hline $25 \mathrm{~A}$ & & $2(7 \%)$ & & & & & & & & \\
\hline $25 \mathrm{~F}$ & & $2(7 \%)$ & & & & & & & & \\
\hline 29 & & & & & & & $\mathrm{I}(<\mathrm{I} \%)$ & & & I (<I\%) \\
\hline $33 \mathrm{~F}$ & & & & & & & $2(2 \%)$ & & & $2(<1 \%)$ \\
\hline $35 \mathrm{~A}$ & & & & & & & $2(2 \%)$ & & & $3(<1 \%)^{*}$ \\
\hline $35 B$ & & & & & & & $\mathrm{I}(<\mathrm{I} \%)$ & & & \\
\hline 38 & & & & & & I (2\%) & $2(2 \%)$ & & & $3(<1 \%)$ \\
\hline 40 & & & & & & & $\mathrm{I}(<\mathrm{l} \%)$ & & & I (<1\%) \\
\hline 41 & & & & & & & & $5(4 \%)$ & & $5(1 \%)$ \\
\hline 45 & & & & & & & $\mathrm{I}(<\mathrm{l} \%)$ & $3(2 \%)$ & & $4(<1 \%)$ \\
\hline 46 & & & & & & & $3(2 \%)$ & $2(2 \%)$ & & $5(<1 \%)$ \\
\hline 48 & & & & & & & & $\mathrm{I}(<\mathrm{I} \%)$ & & I (< | \%) \\
\hline NT & & I (3\%) & & & & & & & & I (<I\%) \\
\hline Total & $18(100 \%)$ & $30(100 \%)$ & 110 (100\%) & 143 (100\%) & $6(100 \%)$ & $64(100 \%)$ & 127 (100\%) & 129 (100\%) & $92(100 \%)$ & $718(100 \%)$ \\
\hline Study & Traore et & & Cadoz et a $\left.\right|^{28}$ & & Leimkuge & et $\mathrm{al}^{29}$ & Enwere et $\mathrm{al}^{30}$ & Onyemelukwe and & Campbell et al ${ }^{32}$ & \\
\hline & & & & & & & Antonio et $\mathrm{al}^{36}$ & Greenwood $^{31}$ & & \\
\hline
\end{tabular}

Notes: Percentages were determined column-wise. *Total for serogroup.

Abbreviations: NT, non-tyepable; $y$, years. 


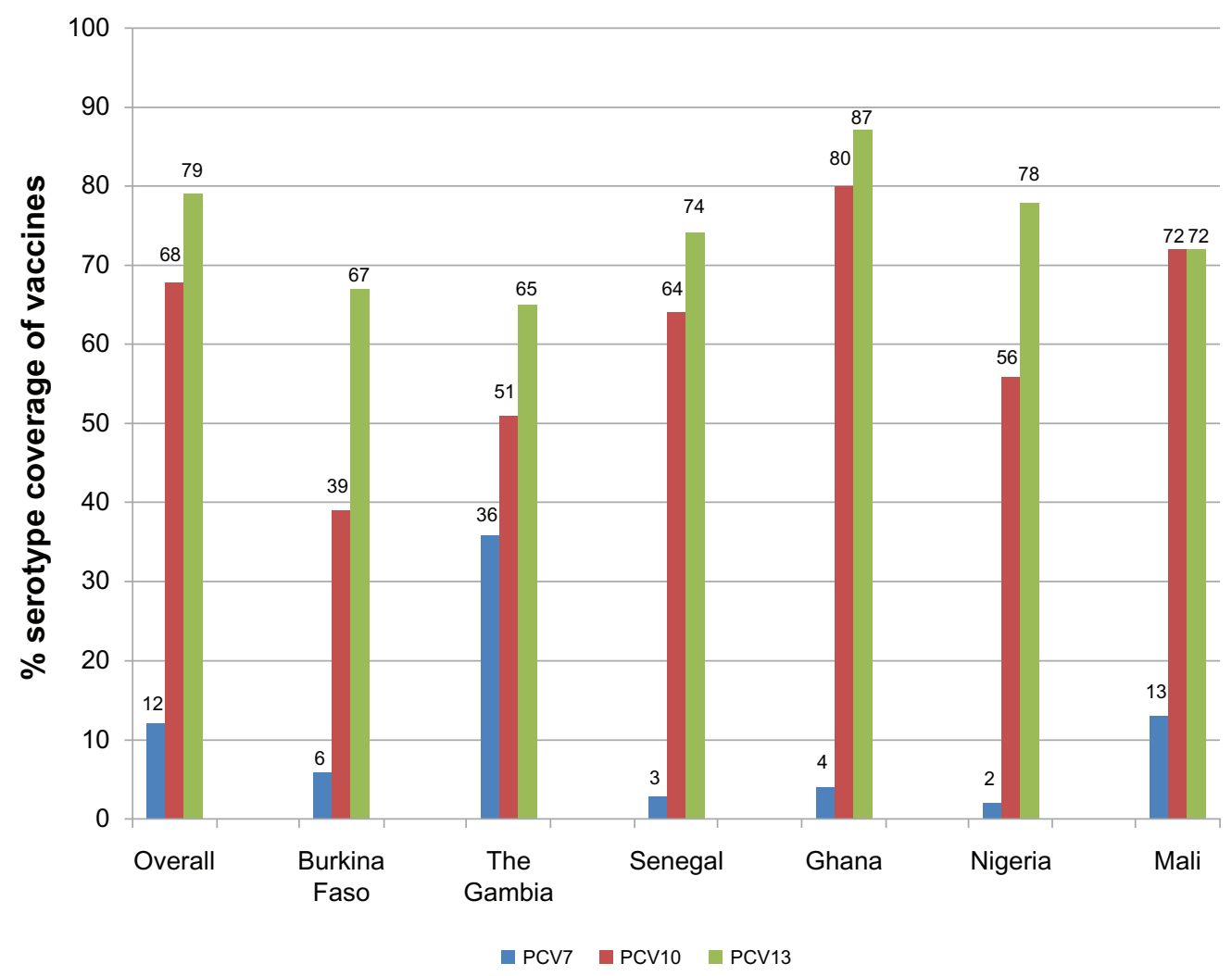

Figure 2 Serotype coverage of pneumococcal conjugate vaccines (PCVs) in various West African countries.

Notes: The serotype compositions of the various conjugate vaccines were 4, 6B, 9V, I4, I8C, I9F, 23F for PCV7; 4, 6B, 9V, I4, I8C, I9F, 23F, I, 5, 7F for PCVI0; and 4, 6B, 9V, 14, 18C, I9F, 23F, I, 5, 7F, 3, 6A, 19A for PCVI3.

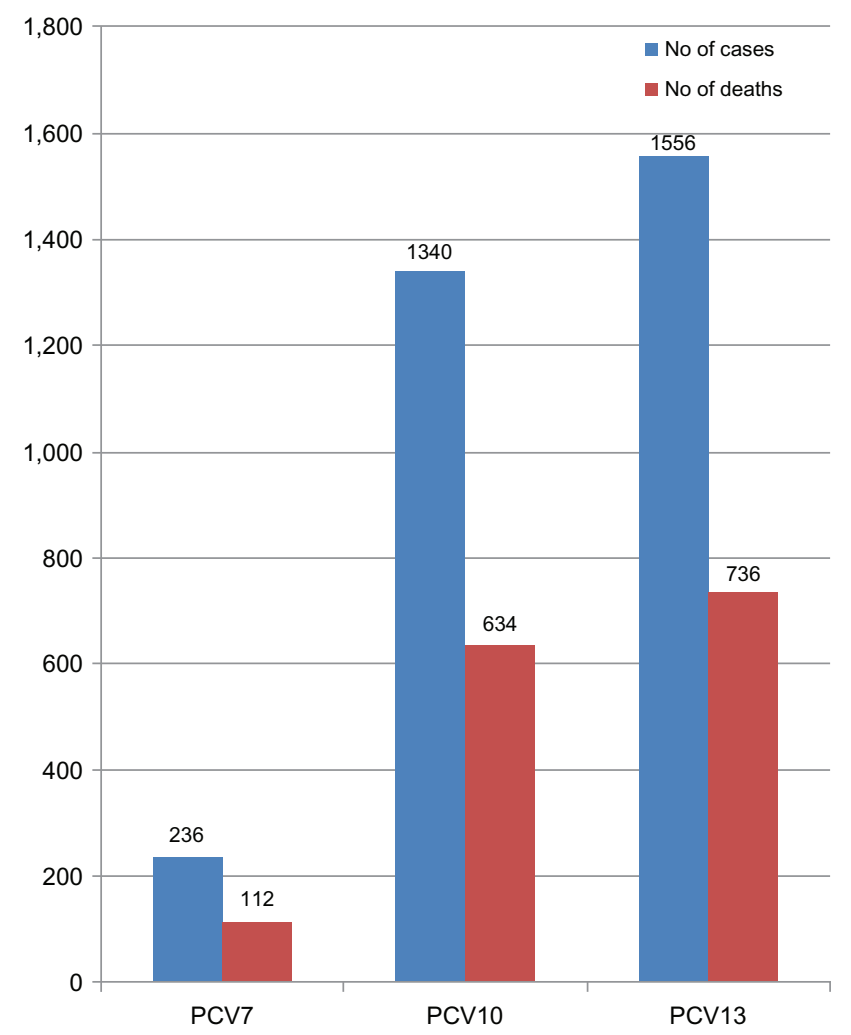

Figure 3 Pneumococcal incidence and mortality cases caused by vaccine serotypes in the West African data.

Abbreviation: PCV, pneumococcal conjugate vaccine. suitable pneumococcal vaccine and in countries in which serotype 3 is important, PCV13 might be the preferred option.

It is important to note that serotype distribution may not be the only factor affecting pneumococcal vaccine choice and that other factors may also be important depending on the context. ${ }^{23,24}$

It is also worth noting from this study that, in most West African countries, $\sim 30 \%$ of serotypes causing invasive disease are not covered by PCV13 (the conjugate vaccine with the highest number of serotypes in current use). Serotypes in this group are more heterogeneous and, epidemiologically, may be difficult to control without vaccination strategies.

Another type of pneumococcal vaccine, the unconjugated polysaccharide vaccine, was the main S.pneumoniae vaccine in use until the development of PCVs. In that vaccine, there are eleven serotypes $(2,8,9 \mathrm{~N}, 10 \mathrm{~A}, 11 \mathrm{~A}, 12 \mathrm{~F}, 15 \mathrm{~F}$, 17F, 20, 22F, and 33F) not included in PCVs. In the West African context, these serotypes make little contribution to pneumococcal disease, so the unconjugated polysaccharide vaccine would probably be of little use in West Africa in the era of PCVs.

In addition to vaccine serotype coverage estimates, this study also provides estimates of pneumococcal disease 
incidence and mortality associated with the serotypes of various PCVs, thus allowing us to project the potential public health impact of the various vaccine formulations in West Africa. However, these data need to be interpreted with caution due to the small number of countries with the requisite data for inclusion in the study. Generally, our data shows that because pneumococcal disease incidence and mortality are so high in West Africa, PCVs (PCV10/ PCV13) could have a tremendous impact in the subregion. Pneumococcal disease is the leading vaccine-preventable disease among children less than 5 years old, and once the appropriate vaccines are made available in the high disease burden region of West Africa, this will contribute to achieving Millennium Development Goal 4, which is to reduce child mortality by two-thirds. ${ }^{1,2,17}$

Although PCVs seem to offer hope in reducing the pneumococcal disease burden, especially in the developing world, where the brunt of the disease burden is borne, potential problems with the vaccines are anticipated. Capsular switching, which has been reported among pneumococci, may lead to evasion of vaccine-conferred immunity. Another potential problem is serotype replacement, where serotypes not contained in the vaccine become relatively more prevalent in disease or carriage as a result vaccination. Serotype replacement has been reported for PCV7 in several industrialized countries such as the $\mathrm{USA}^{25,26}$ and is also expected to occur in the developing world when the vaccines are introduced. Serotype replacement underscores the need for post-vaccine introduction surveillance to monitor the impact of the vaccine and any shifts in the serotype distribution in the area of its use. Additionally, given the limitations of current pneumococcal vaccines, further efforts toward developing improved pneumococcal vaccines should be pursued. In the West African context, perhaps efforts related to the development of conjugate vaccines should target only the serotypes not covered by current pneumococcal vaccines, which are more heterogeneous. Such vaccines would complement the current conjugate vaccines (PCV10 and PCV13) and contribute immensely to the control of invasive pneumococcal disease in the region.

This study has a few limitations. First, pneumococcal serotype distribution may have changed in some of the West African countries over the years, and we recommend a prospective study in the region for comparison with our data. This could also provide useful information on the stability of pneumococcal serotypes in the region. A second limitation of the study is the differences in research design, reagents, and laboratory procedures, including serotyping techniques, used in the different studies included in the systematic review. However, this is less likely to affect the findings of the systematic review, as standard and acceptable protocols were used in the various studies. Thirdly, the nomenclature of a few pneumococcal serotypes (such as serotype 6) has also changed over the years.

\section{Conclusion}

A pneumococcal capsular vaccine for use in West Africa must contain serotypes 1 and 5, which are the most important serotypes causing pneumococcal disease in the region. Consequently, while PCV10 and PCV13 are generally suitable for use in West Africa, PCV7 is unsuitable. Although PCV10 has appreciably high serotype coverage in West Africa generally, it may perform poorly in some West African countries. This is because the three pneumococcal serotypes $(3,6 \mathrm{~A}$, 19A) that are absent in PCV10 but present in PCV13 are important causes of disease in such West African countries.

\section{Acknowledgments}

We thank the authors of the various papers included in this systematic review. Antibiotic Drug use, Monitoring and Evaluation of Resistance (ADMER), a Danish International Development Agency (DANIDA)-sponsored project based at the University of Copenhagen, provided partial funding for the study through the support of the PhD study program of Nicholas TKD Dayie. The funding received is gratefully acknowledged.

\section{Disclosure}

The authors declare no conflicts of interest in this work.

\section{References}

1. Pneumococcal vaccines: World Health Organization position paper. Can Commun Dis Rep. 1999;25(17):150-151. English and French.

2. O'Brien KL, Wolfson LJ, Watt JP, et al; Hib and Pneumococcal Global Burden of Disease Study Team. Burden of disease caused by Streptococcus pneumoniae in children younger than 5 years: global estimates. Lancet. 2009;374(9693):893-902.

3. Van Bambeke F, Reinert RR, Appelbaum PC, Tulkens PM, Peetermans WE. Multidrug-resistant Streptococcus pneumoniae infections: current and future therapeutic options. Drugs. 2007;67(16):2355-2382.

4. Selman S, Hayes D, Perin LA, Hayes WS. Pneumococcal conjugate vaccine for young children. Manag Care. 2000;9(9):49-52, 54, 56-57.

5. Centers for Disease Control and Prevention (CDC). Direct and indirect effects of routine vaccination of children with 7-valent pneumococcal conjugate vaccine on incidence of invasive pneumococcal disease - United States, 1998-2003. MMWR Morb Mortal Wkly Rep. 2005;5(36)4:893-897.

6. Pneumococcal conjugate vaccine for childhood immunization - WHO position paper. Wkly Epidemiol Rec. 2007;82(12):93-104.

7. Greenwood B. Pneumococcal meningitis epidemics in Africa. Clin Infect Dis. 2006;43(6):701-703.

8. Antonio M, Hakeem I, Awine T, et al. Seasonality and outbreak of a predominant Streptococcus pneumoniae serotype 1 clone from The Gambia: expansion of ST217 hypervirulent clonal complex in West Africa. BMC Microbiol. 2008;8:198. 
9. Yaro S, Lourd M, Traoré Y, et al. Epidemiological and molecular characteristics of a highly lethal pneumococcal meningitis epidemic in Burkina Faso. Clin Infect Dis. 2006;43(6):693-700.

10. Leimkugel J, Adams Forgor A, Gagneux S, et al. An outbreak of serotype 1 Streptococcus pneumoniae meningitis in northern Ghana with features that are characteristic of Neisseria meningitidis meningitis epidemics. J Infect Dis. 2005;192(2):192-199.

11. Obaro S, Adegbola R. The pneumococcus: carriage, disease and conjugate vaccines. J Med Microbiol. 2002;51(2):98-104.

12. Hill PC, Akisanya A, Sankareh K, et al. Nasopharyngeal carriage of Streptococcus pneumoniae in Gambian villagers. Clin Infect Dis. 2006;43(6):673-679.

13. Adetifa IM, Antonio M, Okoromah CA, et al. Pre-vaccination nasopharyngeal pneumococcal carriage in a Nigerian population: epidemiology and population biology. PLoS One. 2012;7(1):e30548.

14. Hill PC, Cheung YB, Akisanya A, et al. Nasopharyngeal carriage of Streptococcus pneumoniae in Gambian infants: a longitudinal study. Clin Infect Dis. 2008;46(6):807-814.

15. GAVI Alliance. Pneumococcal vaccine support [web page on the Internet]. Geneva: GAVI Alliance; nd. Available from: http://www. gavialliance.org/support/nvs/pneumococcal/. Accessed on March 20, 2013.

16. Moher D, Liberati A, Tetzlaff J, Altman DG; PRISMA Group. Preferred reporting items for systematic reviews and meta-analyses: the PRISMA statement. PLoS Med. 2009;6(7):e1000097.

17. Johnson HL, Deloria-Knoll M, Levine OS, et al. Systematic evaluation of serotypes causing invasive pneumococcal disease among children under five: the pneumococcal global serotype project. PLoS Med. 2010;7(10). pii: e1000348.

18. Mudhune S, Wamae M; Network Surveillance for Pneumococcal Disease in the East African Region. Report on invasive disease and meningitis due to Haemophilus influenzae and Streptococcus pneumonia from the Network for Surveillance of Pneumococcal Disease in the East African Region. Clin Infect Dis. 2009;48 Supp1 2:S147-S152.

19. Kisakye A, Makumbi I, Nansera D, et al. Surveillance for Streptococcus pneumoniae meningitis in children aged $<5$ years: implications for immunization in Uganda. Clin Infect Dis. 2009;48 Suppl 2: S153-S161.

20. Hoile E. Pneumococcal serotypes in Europe: results of a surveillance report for 1999. Euro Surveill. 2002;6(31):pii 1908.

21. Obaro S. Differences in invasive pneumococcal serotypes. Lancet. 2001;357(9270):1800-1801.

22. GAVI's PneumoADIP. GAVI's Pneumo ADIP: Surveillance and Research Report. Baltimore, MD: GAVI's PneumoADIP; 2007. Available from: http://s3.amazonaws.com/zanran_storage/pneumoadip. idfive.com/ContentPages/2466704508.pdf. Accessed July 8, 2013.

23. Vila-Córcoles A. Advances in pneumococcal vaccines: what are the advantages for the elderly? Drugs Aging. 2007;24(10):791-800.
24. Mathew JL. Pneumococcal vaccination in developing countries: where does science end and commerce begin? Vaccine. 2009;27(32): 4247-4251.

25. Singleton RJ, Hennessy TW, Bulkow LR, et al. Invasive pneumococcal disease caused by nonvaccine serotypes among alaska native children with high levels of 7-valent pneumococcal conjugate vaccine coverage. JAMA. 2007;297(16):1784-1792.

26. Hanage WP. Serotype-specific problems associated with pneumococcal conjugate vaccination. Future Microbiol. 2008;3(1):23-30.

27. Traore Y, Tameklo TA, Njanpop-Lafourcade BM, et al. Incidence, seasonality, age distribution, and mortality of pneumococcal meningitis in Burkina Faso and Togo. Clin Infect Dis. 2009;48 Suppl 2: S181-S189.

28. Cadoz M, Denis F, Mar ID. An epidemiological study of purulent meningitis cases admitted to hospital in Dakar, 1970-1979. Bull World Health Organ. 1981;59(4):575-584. French.

29. Leimkugel J, Adams Forgor A, Gagneux S, et al. An outbreak of serotype 1 Streptococcus pneumoniae meningitis in northern Ghana with features that are characteristic of Neisseria meningitidis meningitis epidemics. J Infect Dis. 2005;192(2):192-199.

30. Enwere G, Biney E, Cheung Y, et al. Epidemiologic and clinical characteristics of community-acquired invasive bacterial infections in children aged 2-29 months in The Gambia. Pediatr Infect Dis J. 2006;25(8):700-705.

31. Onyemelukwe GC, Greenwood BM. Pneumococcal serotypes, epidemiological factors and vaccine strategy in Nigerian patients. J Infect. 1983;5(2):157-163.

32. Campbell JD, Kotloff KL, Sow SO, et al. Invasive pneumococcal infections among hospitalized children in Bamako, Mali. Pediatr Infect Dis J. 2004;23(7):642-649.

33. Adegbola RA, Hill PC, Secka O, et al. Serotype and antimicrobial susceptibility patterns of isolates of Streptococcus pneumoniae causing invasive disease in The Gambia 1996-2003. Trop Med Int Health. 2006;11(7):1128-1135.

34. Antonio M, Hakeem I, Awine T, et al. Seasonality and outbreak of a predominant Streptococcus pneumoniae serotype 1 clone from The Gambia: expansion of ST217 hypervirulent clonal complex in West Africa. BMC Microbiol. 2008;8:198.

35. Yaro S, Lourd M, Traoré Y, et al. Epidemiological and molecular characteristics of a highly lethal pneumococcal meningitis epidemic in Burkina Faso. Clin Infect Dis. 2006;43(6):693-700.

36. Antonio M, Dada-Adegbola H, Biney E, et al. Molecular epidemiology of pneumococci obtained from Gambian children aged 2-29 months with invasive pneumococcal disease during a trial of a 9-valent pneumococcal conjugate vaccine. BMC Infect Dis. 2008;8:81.
International Journal of General Medicine

\section{Publish your work in this journal}

The International Journal of General Medicine is an international, peer-reviewed open-access journal that focuses on general and internal medicine, pathogenesis, epidemiology, diagnosis, monitoring and treatment protocols. The journal is characterized by the rapid reporting of reviews, original research and clinical studies across all disease areas.

\section{Dovepress}

A key focus is the elucidation of disease processes and management protocols resulting in improved outcomes for the patient.The manuscript management system is completely online and includes a very quick and fair peer-review system. Visit http://www.dovepress.com testimonials.php to read real quotes from published authors. 